\title{
А.С. Аникин
}

\section{Изменчивость импульсных реакций сухопутных трасс распространения сантиметровых радиоволн в десятисекундных интервалах}

Приведены экспериментальные оценки усреднённых импульсных реакций для различных сухопутных трасс распространения радиоволн прямой видимости и их статистические характеристики для различных угловых положений антенны источника радиоизлучения.

Ключевые слова: экспериментальные данные, импульсная характеристика, многолучевость, фильтр Винера, статистические характеристики, угловое положение антенны, источник радиоизлучения.

doi: $10.21293 / 1818-0442-2017-20-2-10-14$

Известно, что искажения выходных сигналов приёмных антенн при распространении радиоволн на сухопутных наземных трассах открытого и закрытого типов вызваны, главным образом, многолучевостью $[1,2]$. Многолучевость связана с отражением радиоволн от подстилающей поверхности и находящимися на ней объектами (местными предметами). Это явление приводит к снижению точности измерения радиотехнических параметров принятых сигналов и к ухудшению технических характеристик пассивных радиотехнических систем мониторинга $[3,4]$. Для повышения точности измерения параметров искажённых сигналов необходимо априорное знание характера многолучевого распространения радиоволн на наземных трассах [3].

Наглядно явление многолучевости описывает импульсная реакция радиоканала, которая, как известно, является вещественной случайной функцией времени, описывающей отклик эквивалентной линейной системы на воздействие в виде дельтаимпульса $[1,2,4]$. Для коротких наземных трасс с выраженным отражением радиоволн объектами рельефа местности модель импульсной характеристики представляют суммой задержанных дельтафункций, представляя объекты на местности моделью точечных излучателей $[1,2]$ :

$$
h(\tau)=\sum_{i=1}^{N} a_{i} \delta\left(\tau-\tau_{i}\right),
$$

где $N$ - количество отражённых сигналов; $a_{i}-$ весовой коэффициент $i$-го отражённого сигнала; $\tau_{i}-$ задержка $i$-го отражённого сигнала относительно момента прихода сигнала по кратчайшему пути. Отражённому сигналу от точечного объекта соответствует пик импульсной реакции.

Так как импульсная реакция наземной трассы имеет случайный характер, то для её описания используют статистические характеристики [4].

Анализ доступных литературных источников показал, что существует внушительный объём работ как по оценкам импульсных реакций наземного канала распространения, так и по оценкам их статистических характеристик. В частности, для проектирования и моделирования систем радиосвязи разра- ботаны модели радиоканалов, в основном, для зоны городской застройки, которые представляют собой таблицы с количеством, задержками и ослаблениями отражённых сигналов [5-7].

Для пассивных радиотехнических средств мониторинга типична ситуация, когда имеется многоканальная приёмная аппаратура, а остронаправленная антенна источника радиоизлучения ориентирована не на пункт приёма. К сожалению, оценки импульсных реакций сухопутной трассы распространения при изменении углового положения остронаправленной антенны передатчика исследованы недостаточно глубоко. Так, в работе [6] приводятся лишь усреднённые импульсные реакции для разной ориентации передающей антенны, и никаких оценок статистических характеристик при этом не делается. В статье [7] приводятся сведения о коэффициенте корреляции сигналов, разнесённых по времени, только для тропосферного радиоканала. В системах радиосвязи с пространственно-временным кодированием используется передаточная функция радиоканала в виде матрицы. Однако результаты исследования её статистических характеристик или связанных с ней импульсных реакций для сухопутных трасс, к сожалению, не найдены.

Цель работы - представить статистические характеристики импульсной реакции сухопутных трасс распространения радиоволн для пространственно разнесённых приёмных антенн при различной ориентации остронаправленной антенны передатчика относительно приёмного пункта.

Статистические характеристики определены путём обработки экспериментальных данных, полученных сотрудниками НИИ РТС ТУСУРа $[8,9]$.

На оценку импульсной реакции канала распространения радиоволн влияют характеристики экспериментальной аппаратуры, поэтому приведём её краткое описание.

Описание экспериментальной установки

Для проведения экспериментальных измерений использовались приёмно-измерительная аппаратура (приёмный пункт) и аппаратура передающего пункта (РЛС), изготовленная сотрудниками НИИ РТС ТУСУРа. Подробное описание цифровой приёмноизмерительной аппаратуры изложено в работе [9]. 
Во время проведения экспериментальных измерений антенна РЛС принимала фиксированные угловые положения в интервале $\pm 6^{\circ}$ с шагом $3^{\circ}$, отсчитываемые от направления на приёмный пункт.

Аппаратуры источника излучения и приёмного пункта были синхронизированы. При измерениях производилась запись в память ЭВМ цифровых отсчётов принятых сигналов за определённый промежуток времени (в окне регистрации). Укрупнённая структурная схема комплекса при проведении экспериментальных измерений на наземных трассах прямой видимости и в зоне дифракции приведена на рис. 1 .

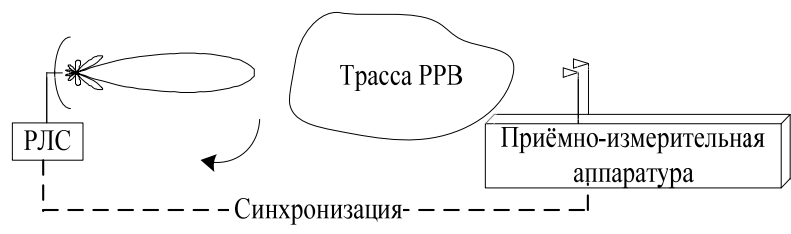

Рис. 1. Структурная схема экспериментального комплекса

Оценка импульсных реакций сухопутной трассы распространения радиоволн производилась по импульсным радиосигналам радиопередающего комплекса (РПК-1) сантиметрового диапазона длительностью 250 нс (по уровню -3 дБ) и частотой повторения 2 кГц. Радиолокационная станция РПК-1 имеет зеркально-параболическую антенну диаметром 110 см типа Кассегрена с облучателем в виде открытого конца прямоугольного волновода и сигарообразной формой диаграммы направленности (ДНА). Ширина ДНА в плоскости азимута по уровню -3 дБ около $1,7^{\circ}$. Уровень боковых лепестков не превышает -15 дБ. При обработке экспериментальных данных рассматривались только случаи, когда антенна передатчика излучала радиоволну вертикальной поляризации.

Приёмник с динамическим диапазоном 71 дБ и полосой пропускания 40 МГц имеет чувствительность -100 дБ/мВт (3,2 мкВ). Перед аналогоцифровым преобразованием сигнал переносится на видеочастоту квадратурным демодулятором.

Антенная система приёмного пункта (рис. 2), состоящая из восьми прямоугольных рупоров, расположенных в два ряда, наводилась на источник излучения по максимуму сигнала. Ширина диаграммы направленности рупорной антенны в горизонтальной плоскости по уровню -3 дБ около $8,5^{\circ}$.

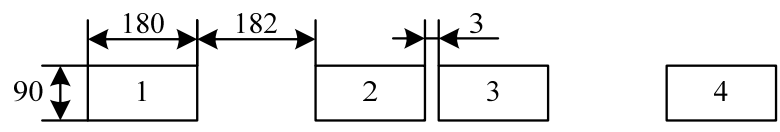

Рис. 2. Внешний вид и размеры антенной системы при измерениях (заимствовано из [9])

Антенны вертикальной поляризации имеют нечётный номер, антенны горизонтальной поляризации имеют чётный номер. Отношение сигнал/шум при оценке импульсных характеристик составляло не менее 30 дБ.
Метод оценки импульсной реакции

Импульсные реакции сухопутных наземных трасс распространения радиоволн оценивались методом Винера с помощью обратного преобразования Фурье передаточной функции канала распространения $\hat{\dot{K}}(\omega)$, которая определяется выражением [10]

$$
\hat{\dot{K}}(\omega)=\frac{\dot{S}_{\text {изл }}^{*}(\omega)\left[\dot{S}_{\text {изл }}(\omega) \dot{K}(\omega)+\dot{N}(\omega)\right]}{\left|\dot{S}_{\text {изл }}(\omega)\right|^{2}+G_{N}(\omega)},
$$

где $\dot{S}_{\text {пр }}(\omega)=\dot{S}_{\text {изл }}(\omega) \dot{K}(\omega)+\dot{N}(\omega)-$ преобразование Фурье от реализации принятого сигнала на интервале наблюдения длительностью $T_{n} ; \dot{S}_{\text {изл }}(\omega)$ - преобразование Фурье от реализации излученного сигнала; $\dot{N}(\omega)$ - преобразование Фурье от реализации шума приёмной аппаратуры; $\dot{K}(\omega)$ - передаточная функция канала распространения в полосе сигнала; $G_{N}(\omega)=|\dot{N}(\omega)|^{2}$ - энергетический спектр шума приёмной аппаратуры [11].

При оценке импульсной характеристики сухопутных трасс распространения радиоволн с помощью (2) считалось, что последовательность отсчётов импульсной реакции в равноотстоящие моменты относительно времени приёма периодических импульсных сигналов представляет собой выборку из стационарного случайного процесса. При отсутствии шума ( $\dot{N}(\omega)=0$ и $G_{N}(\omega)=0$ ) формула (2) сводится к передаточной функции канала распространения:

$$
\hat{\dot{K}}(\omega)=\dot{S}_{\text {изл }}^{*}(\omega) \dot{S}_{\text {изл }}(\omega) \dot{K}(\omega) /\left|\dot{S}_{\text {изл }}(\omega)\right|^{2}=\dot{K}(\omega) .
$$

\section{Проверка метода оценки импульсной} реакции моделированием

При оценке импульсной реакции канала распространения по экспериментальным данным с помощью выражения (2) использовались принятые сигналы в виде дискретных отсчётов в результате аналого-цифрового преобразования. Для применения выражения (2) вместо классического преобразования Фурье необходимо использовать дискретное преобразование Фурье. Для проверки корректности замены классического преобразования Фурье дискретным при использовании выражения (2) проведено цифровое моделирование. Форма и длительность зондирующего сигнала задавались, как для излучаемого сигнала РПК-1, аддитивный шум приёмной аппаратуры представлялся нормальным случайным процессом. Задавалась исходная импульсная характеристика, содержащая три пика с задержками $0,2,0,25$ и 0,5 мкс и относительными амплитудами $1 ; 0,7$ и 0,4 соответственно. Принятый сигнал моделировался путём свёртки зондирующего сигнала с исходной импульсной характеристикой, после чего к результату свёртки добавлялся нормальный случайный процесс. Отношение сигнал/шум задавалось равным 20 дБ, что соответствовало условиям прове- 
дения эксперимента. Результат оценки импульсной реакции трассы распространения по формуле (2) показан на рис. 3.

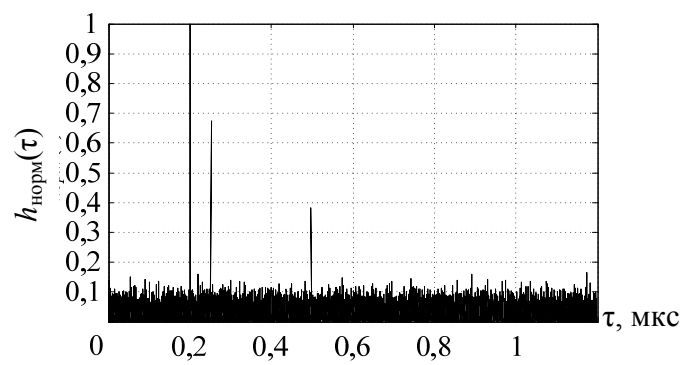

Рис. 3. Пример результата оценки нормированной импульсной реакции радиоканала

Анализ показал, что временное положение пиков импульсной реакции определяется с ошибкой не более $\sigma_{\tau}<20$ нс при наблюдении принятого сигнала на интервале длительностью не меньше 1200 мкс, а амплитуды пиков определяются с точностью не хуже $10 \%$ при отношении сигнал/шум не меньше 15 дБ.

\section{Статистические характеристики}

\section{импульсной реакции сухопутной трассы} распространения радиоволн

Оценки импульсных реакций производились для сухопутных открытых полузакрытых трасс протяженностью от 16 до 30 км. Наличие отражённых радиоволн на таких трассах обусловлено преимущественно элементами рельефа местности: подстилающей поверхностью, объектами на местности природного и искусственного происхождения, кромкой лесных массивов и т.п.

Учитывался вклад в импульсную реакцию только наиболее существенных отражённых сигналов: определялся пик с максимальной амплитудой в данной реализации, а остальные пики определялись по превышению их амплитуд порога $30 \%$ от максимального уровня пика.

По экспериментальным данным были построены гистограммы амплитуд пиков и определены:

- усреднённая по совокупности реализаций импульсная реакция $E(\tau)$;

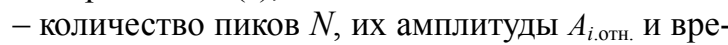
мя прихода относительно момента превышения первым пиком порога 30 \%;

- СКО флуктуации амплитуд пиков $\sigma_{i}$ импульсной реакции;

- коэффициенты корреляции флуктуаций амплитуд пиков для одной антенны и флуктуаций амплитуд пиков разных приёмных антенн.

Примеры усреднённой импульсной реакции для сухопутных трасс протяжённостью 16 и 28 км при ориентации антенны передатчика в направлении на приёмный пункт приведены на рис. 4 и 5, соответственно.

Из этих рисунков видно, что длительность импульсной реакции сухопутных открытых трасс до 30 км определяется главным образом расположением объектов на местности и в меньшей степени протяжённостью трассы.

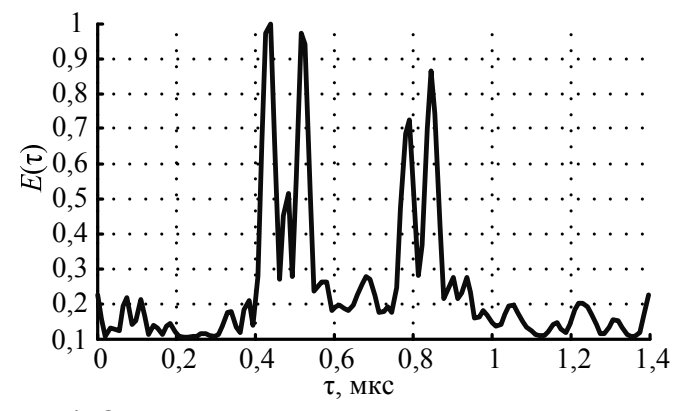

Рис. 4. Оценка нормированной импульсной реакции радиоканала для сухопутной открытой трассы протяжённостью 16 км

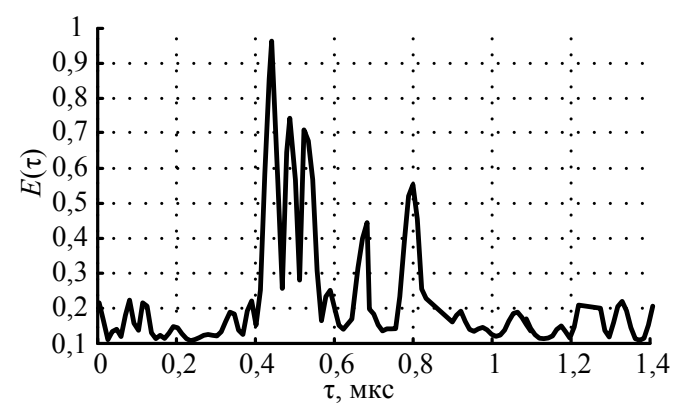

Рис. 5. Нормированная оценка импульсной реакции радиоканала для сухопутной открытой трассы протяжённостью 28 км

Отворот антенны передатчика от направления на приёмный пункт приводит к перераспределению амплитуд отражённых сигналов, причем первый пик, как правило, имеет меньшую амплитуду в сравнении с остальными отражёнными сигналами (рис. 6). Меняется также и время прихода отражённых сигналов относительно момента превышения первым пиком импульсной реакции порога $30 \%$.

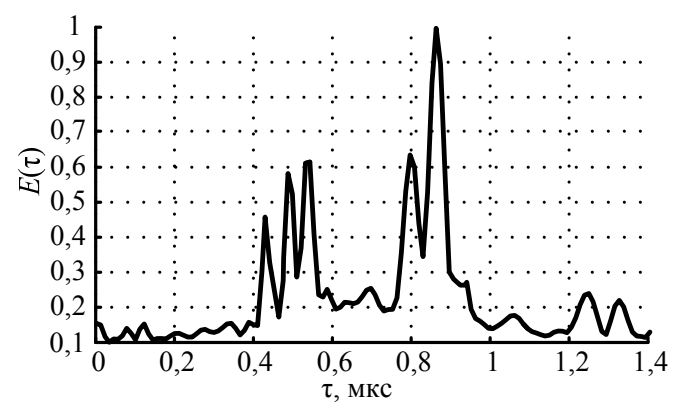

Рис. 6. Нормированная оценка импульсной реакции радиоканала для сухопутной открытой трассы протяжённостью 28 км при отвороте антенны передатчика на $6^{\circ}$ относительно приёмного пункта

Статистические характеристики импульсных реакций для указанных трасс при ориентации антенны передатчика в направлении на приёмный пункт представлены в табл. 1 и 2, а в табл. 3 - при отвороте передающей антенны на $6^{\circ}$ от направления на приёмный пункт.

В табл. 1-3 указаны: $N$ - номер пика; $A_{i . \text { отн }}-$ амплитуда $i$-го пика, относительно максимальной амплитуды пика импульсной реакции; $\tau_{i}-$ задержка $i$-го 
пика относительно момента первого пика; $\sigma_{i}$ - среднеквадратическое значение флуктуаций $i$-го пика импульсной реакции.

Т а бли ц а 1 Характеристики усреднённой импульсной реакции сухопутной открытой трассы протяженностью 16 км при ориентации передающей антенны в направлении на приёмный пункт

\begin{tabular}{|c|c|c|c|c|c|}
\hline$N$ & 1 & 2 & 3 & 4 & 5 \\
\hline$A_{\text {іотн }}$ & 1 & 0,51 & 0,97 & 0,72 & 0,86 \\
\hline$\tau_{\mathrm{j}}$, мкс & 0 & 0,04 & 0,08 & 0,35 & 0,41 \\
\hline$\sigma_{i}, \mathrm{MB}$ & 3 & 24 & 29 & 27 & 31 \\
\hline
\end{tabular}

Таблица 2

Характеристики усреднённой импульсной реакции сухопутной открытой трассы протяженностью 28 км при ориентации передающей антенны в направлении на приёмный пункт

\begin{tabular}{|c|c|c|c|c|c|}
\hline \multicolumn{7}{|c|}{ на приёмный пункт } \\
\hline$A_{i \text { отн }}$ & 1 & 2 & 3 & 4 & 5 \\
\hline$\tau_{i}$, мкс & 0 & 0,72 & 0,68 & 0,45 & 0,55 \\
\hline$\sigma_{i}, \mathrm{MB}$ & 2 & 34 & 20 & 27 & 41 \\
\hline
\end{tabular}

Т а бл и ц а 3

Характеристики усреднённой импульсной реакции сухопутной открытой трассы протяженностью 28 км при отвороте передающей антенны на $6^{\circ}$ от направления на приёмный пункт

\begin{tabular}{|c|c|c|c|c|c|}
\hline$N$ & 1 & 2 & 3 & 4 & 5 \\
\hline$A_{i \text { отн }}$ & 0,48 & 0,59 & 0,62 & 0,64 & 1 \\
\hline$\tau_{i}$, мкс & 0 & 0,04 & 0,1 & 0,35 & 0,53 \\
\hline$\sigma_{i}, \mathrm{MB}$ & 2,5 & 38 & 42 & 51 & 65 \\
\hline
\end{tabular}

Как видно из таблиц флуктуации амплитуд отражённых волн в десятки раз больше, чем флуктуации амплитуды пика, пришедшего по кратчайшему пути. Анализ результатов обработки экспериментальных данных для совокупности указанных трасс распространения и различных угловых положений антенны передатчика показывает, что отношение среднего значения амплитуды пика к среднеквадратическому отклонению флуктуаций амплитуды пика составляет от 2 до 30. Задержки отражённых сигналов с амплитудами, превышающими 30\% относительно пика, пришедшего по кратчайшему пути, достигают 0,7 мкс.

Для примера на рис. 7 приведена гистограмма флуктуаций амплитуды пика №2 (с задержкой $\tau_{2}$ ) для усреднённой импульсной реакции, изображённой на рис. 4.

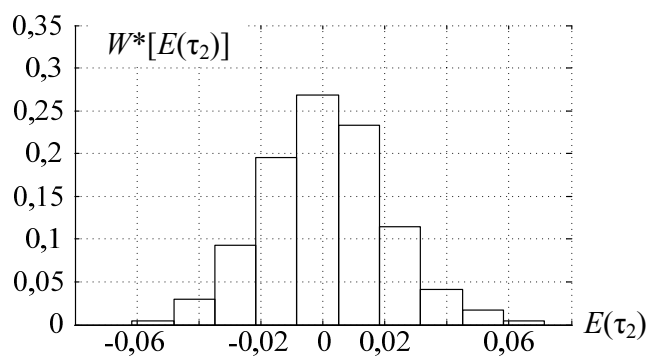

Рис. 7. Гистограмма флуктуаций амплитуды второго пика для сухопутной открытой трассы протяжённостью 16 км при ориентации антенны передатчика в направлении на приёмный пункт
Аналогичные гистограммы получены и для других пиков. В 95\% случаев флуктуации амплитуд лучей отражённых сигналов не противоречат нормальному закону распределения по критерию Хи-квадрат по уровню значимости 5\%.

Совместно с законом распределения оценивалась глубина флуктуаций амплитуды пика как разница между минимальной и максимальной амплитудой пика по совокупности импульсных реакций. Глубокие замирания амплитуды пика и отличие закона распределения его флуктуаций от нормального закона свидетельствуют о том, что данному пику соответствует интерференция сигналов не более чем от 6 источников отражений. Напротив, обобщённый закон распределения Рэлея для флуктуаций амплитуды пика и глубина замирания амплитуд менее 13,4 дБ свидетельствуют о большом количестве $(N \gg 10)$ источников отражений.

Оценим корреляцию амплитуд лучей импульсной реакции для сухопутной трассы протяжённостью 16 км. Оценки коэффициентов корреляции флуктуации амплитуд 5 пиков импульсной реакции для $i$-й антенны записывались в виде матрицы $\mathbf{R}_{i}$ вида:

$$
\mathbf{R}_{i}=\left[\begin{array}{ccccc}
r_{11}^{i} & r_{12}^{i} & r_{13}^{i} & r_{14}^{i} & r_{15}^{i} \\
r_{21}^{i} & r_{22}^{i} & r_{23}^{i} & r_{24}^{i} & r_{25}^{i} \\
r_{31}^{i} & r_{32}^{i} & r_{33}^{i} & r_{34}^{i} & r_{35}^{i} \\
r_{41}^{i} & r_{42}^{i} & r_{43}^{i} & r_{44}^{i} & r_{45}^{i} \\
r_{51}^{i} & r_{52}^{i} & r_{53}^{i} & r_{54}^{i} & r_{55}^{i}
\end{array}\right],
$$

где $r_{k l}^{i}$ - коэффициент корреляции флуктуаций амплитуд $k$-го и $l$-го отражённых сигналов $i$-й антенны.

Оценки коэффициентов корреляций флуктуации амплитуд 5 пиков импульсной реакции для $i$-й и $j$-й пространственно разнесённых антенн (см. рис. 2) записывались в виде матрицы $\mathbf{R}_{i j}$ вида

$$
\mathbf{R}_{i j}=\left[\begin{array}{rrrrr}
r_{11}^{i j} & r_{21}^{i j} & r_{31}^{i j} & r_{41}^{i j} & r_{51}^{i j} \\
r_{12}^{i j} & r_{22}^{i j} & r_{32}^{i j} & r_{42}^{i j} & r_{52}^{i j} \\
r_{13}^{i j} & r_{23}^{i j} & r_{33}^{i j} & r_{43}^{i j} & r_{53}^{i j} \\
r_{14}^{i j} & r_{24}^{i j} & r_{34}^{i j} & r_{44}^{i j} & r_{54}^{i j} \\
r_{15}^{i j} & r_{25}^{i j} & r_{35}^{i j} & r_{45}^{i j} & r_{55}^{i j}
\end{array}\right],
$$

где $r_{k l}^{i j}$ - коэффициент корреляции флуктуаций амплитуд $k$-го и $l$-го пиков $i$-й и $j$-й антенн.

Примеры указанных корреляционных матриц для сухопутной трассы протяжённостью 16 км приведены ниже:

$$
\begin{aligned}
\mathbf{R}_{1} & =\left[\begin{array}{ccccc}
1 & 0,71 & 0,16 & 0,17 & 0,1 \\
0,71 & 1 & 0,23 & 0,08 & 0,08 \\
0,16 & 0,23 & 1 & 0,04 & 0,22 \\
0,17 & 0,08 & 0,04 & 1 & 0,76 \\
0,1 & 0,08 & 0,22 & 0,76 & 1
\end{array}\right], \\
\mathbf{R}_{13} & =\left[\begin{array}{ccccc}
0,36 & 0,26 & 0,13 & 0,22 & 0,11 \\
0,3 & 0,33 & 0,02 & 0,15 & 0,08 \\
0,25 & 0,25 & 0,01 & 0,06 & 0,15 \\
0,12 & 0,08 & 0,09 & 0,34 & 0,28 \\
0,1 & 0,07 & 0,18 & 0,01 & 0,19
\end{array}\right] .
\end{aligned}
$$


Анализ корреляционных матриц показал, что в $85 \%$ случаев коэффициент корреляции амплитуд отражённых сигналов ниже 0,3. Амплитуды отражённых сигналов практически не коррелированы при разносе приёмных пунктов более $6 \lambda$, достигая в единичных случаях значения 0,5 .

Аналогичные результаты были получены и для других наземных трасс открытого и закрытого типа протяженностью до 30 км. К сожалению, в рамках статьи представить статистические характеристики для других сухопутных трасс не представляется возможным.

\section{Выводы}

Результаты обработки усреднённых импульсных реакций позволяют сделать следующие выводы для исследованных сухопутных трасс распространения:

1. Усреднённая по совокупности реализаций импульсная реакция содержит, как правило, счётное количество отдельных пиков, соответствующих отражённым от препятствий сигналам. Количество отражённых сигналов с вероятностью $85 \%$ находится в интервале от 4 до 10 при условии, что их амплитуды выше 30\% от амплитуды сигнала, пришедшего по кратчайшему пути.

2. По глубине флуктуаций амплитуды отражённого сигнала можно дать грубую оценку количества отражателей («блестящих» точек).

3. Отворот узконаправленной антенны передатчика от направления на приёмный пункт в секторе $\pm 6^{\circ}$ приводит к случайному изменению амплитуд отражённых сигналов и их задержек относительно сигнала, пришедшего по кратчайшему пути.

4. Отношение средней амплитуды отражённого сигнала к его среднеквадратическому значению с вероятностью $72 \%$ находится в интервале от 2 до 30 . В среднем задержка отражённого сигнала с амплитудой более $30 \%$ от амплитуды сигнала, пришедшего по кратчайшему пути, достигает 0,5 мкс.

5. Распределение амплитуд отражённых сигналов в 95\% случаев не противоречит нормальному закону.

6. Коэффициент корреляции флуктуаций амплитуд отражённых сигналов с вероятностью $98 \%$ составляет от 0,05 до 0,8, в зависимости от трассы распространения (от 16 до 30 км) и отворота антенны передатчика. Амплитуды отражённых сигналов для приёмных пунктов с разнесением более $6 \lambda$ в большинстве случаев можно считать некоррелированными.

Исследование выполнено при финансовой поддержке РФФИ в рамках научного проекта 16-3860091 мол_а_дк.

\section{Лuтература}

1. Быков В.В. Цифровое моделирование в статистической радиотехнике. - М.: Советское радио, 1971. - 328 с.
2. Кеннеди Р. Каналы связи с замираниями и рассеянием. - М.: Советское радио, 1973 . - 302 с.

3. Радиомониторинг - задачи, методы, средства / под ред. А.М. Рембовского. - 2-е изд., перераб. и доп. - М.: Горячая линия-Телеком, 2010. -624 c.

4. Bello P.A. Characterization of randomly time-variant linear channels // IEEE Transactions on communications systems. - 1963. - Vol. 11, No. 4. - P. 360-393.

5. Рекомендация МСЭ-R P.1816-2 Прогнозирование пространственно-временного профиля для широкополосных сухопутных подвижных служб. - Сер. Р, 09/2013.

6. Ворошилин Е.П. Экспериментальная оценка импульсной реакции канала распространения радиоволн в сантиметровом диапазоне / Е.П. Ворошилин, В.Ю. Лебедев // Доклады ТУСУРа. - 2008. - № 2. - С. 5-9.

7. Результаты экспериментальных исследований энергетической импульсной реакции тропосферного канала / В.А. Филоненко, В.В. Ручкин, Е.Д. Тельпуховский, В.Б Фортес // Изв. вузов. Радиофизика. - 1976. - Т. 19, № 7. - C. 984-992.

8. Вершинин А.С. Экспериментальная оценка импульсной реакции канала распространения радиоволн в сантиметровом диапазоне / А.С. Вершинин, Д.А. Коротков, Е.П. Ворошилин // Доклады ТУСУРа. - 2011. - № 2. C. $78-84$.

9. Отчёт по НИР «Пространственно-временные модели ультракоротковолновых сигналов, распространяющихся вдоль неровной земной поверхности». - Томск: ТУСУР. $-2008 .-250 \mathrm{c}$.

10. Оппенгейм Э. Применение цифровой обработки сигналов. - М.: Мир, 1980. - 550 с.

11. Каратаева Н.А. Радиотехнические цепи и сигналы: учеб. пособие. - Ч. 1.-Томск, ТУСУР. - 2001. $260 \mathrm{c}$.

12. Сифоров В.И. Моделирование многолучевых радиоканалов для анализа и синтеза систем передачи информации / В.И. Сифоров, А.В. Просин. - М.: Наука, 1978. - $168 \mathrm{c}$.

\section{Аникин Алексей Сергеевич}

Канд. техн. наук, ассистент

каф. радиотехнических систем ТУСУРа

Тел.: 8 (382-2) 41-38-98

Эл. почта: rrb86@inbox.ru

Anikin A.S.

Impulse responses variability of the terrestrial paths of propagation of centimeter radio waves in ten-second intervals

Experimental evaluation of energy impulse responses for different land propagation paths line of sight and their statistical characteristics for different angular positions of the antenna of the radio source is described.

Keywords: experimental data, impulse response, multipath, Wiener filter, statistical characteristics, angular position of antenna, radio emission source. 